Published in final edited form as:

Trends Mol Med. 2018 June ; 24(6): 522-524. doi:10.1016/j.molmed.2018.04.007.

\title{
To scar or not to scar
}

\author{
Aarti Narang and Binhai Zheng ${ }^{\star}$ \\ Department of Neuroscience, University of California San Diego, La Jolla, California, USA
}

\begin{abstract}
A recent study indicates that reducing fibrotic scarring by genetically abrogating the proliferation of type A pericytes promotes axon regeneration and functional recovery after spinal cord injury. Questions remain regarding the identity of the cells being manipulated and the balance between the beneficial and detrimental effects of fibrotic scarring.
\end{abstract}

\section{Keywords}

fibrotic scar; astrocytic scar; spinal cord injury; axon regeneration; functional recovery

Spinal cord injury is known to trigger a series of events that mediate wound closure by recruitment of local and infiltrating immune cells, extracellular matrix-producing fibroblasts/ pericytes and astrocytes that wall off the non-neural lesion core forming a scar. Scar is an overarching term that describes an astrocytic component and a non-neural fibrotic component of fibroblasts/pericytes, ECM proteins, and infiltrating macrophages and other blood-borne cells (Figure 1). Although the astrocytic component of the scar has been the subject of intensive focus in the past, the contribution of the fibrotic component of the scar has gained increasing attention.

In a recent issue of Cell, Dias and colleagues reported that attenuating pericyte-derived fibrotic scarring promotes axon regeneration and functional recovery after spinal cord injury [1]. Their latest study is a follow-up of their previous study suggesting that a specific subset of pericytes (called type A pericytes) proliferate, leave the blood vessel wall, and differentiate into scar-forming stromal cells that make up much of the fibrotic scar [2]. As in their previous study [2], the researchers used an inducible Cre transgene under the promoter of the sodium-dependent glutamate/aspartate transporter gene (Glast) to mark type A pericytes in mice with a ROSA26-YFP reporter and to simultaneously eliminate all Ras activity in these cells to prevent their proliferation, thereby reducing fibrotic scarring. They observed a dose (Cre recombination efficiency) dependent reduction of fibrotic scarring that was accompanied by enhanced corticospinal and serotonergic axon regeneration/sprouting, functional integration of regenerated CST axons and improved behavioral recovery,

*Correspondence (binhai@ucsd.edu).

Publisher's Disclaimer: This is a PDF file of an unedited manuscript that has been accepted for publication. As a service to our customers we are providing this early version of the manuscript. The manuscript will undergo copyediting, typesetting, and review of the resulting proof before it is published in its final citable form. Please note that during the production process errors may be discovered which could affect the content, and all legal disclaimers that apply to the journal pertain. 
implicating a key role for type A pericytes in modulating anatomical and functional repair following CNS injury.

Assuming that these results can be independently verified, the study by Dias and colleagues provides the strongest evidence to date on the role of fibrotic scarring in restricting axonal growth and functional recovery after spinal cord injury. Earlier studies modifying the ECM associated with the fibrotic scar provided suggestive evidence [3]. More recently, pharmacological inhibition of fibrotic scarring with microtubule-stabilizing agents has been shown to promote axon regeneration and functional recovery after spinal cord injury [4]. However, these microtubule-stabilizing agents also act directly on neurons to influence regeneration, thus the effect of manipulating fibrotic scarring alone remains to be clarified. The elegance of the Dias and colleagues' study lies in the use of a complex genetic scheme, also applied in their previous study [2], that combines five genetic modifications (targeted mutations or transgenes) into one experimental mouse line (Glast-CreER ${ }^{\mathrm{T} 2}, \mathrm{HRas}^{-/-}$, NRas ${ }^{-/}, \mathrm{KRas}^{\mathrm{f} / \mathrm{f}}$, ROSA26-YFP) [1]. This allowed the authors to specifically delete KRas and consequently eliminate all Ras activity in Glast-CreER ${ }^{\mathrm{T} 2}$-expressing cells upon tamoxifen induction without directly affecting neurons. Eliminating Ras activity inhibits type A pericyte proliferation and reduces fibrotic scarring rather than killing existing pericytes, which have important physiological functions such as supporting the blood brain barrier. Overall, this study depicts the tantalizing possibility that modulating type A pericyte proliferation could develop into a promising strategy to promote repair and recovery after spinal cord injury.

Important questions remain. The use of a Glast promoter-based transgene to mark and manipulate type A pericytes remains perplexing given that Glast is also expressed in other cell types - including astrocytes. Indeed, the exact same transgene used in this study was originally developed by the authors to conduct gene manipulation in astrocytes [5]. It is not clear how the specificity of this transgene for astrocytes has apparently shifted to type A pericytes in spinal cord injury models. Because reactive astrocytes impact CNS repair in a major way and represent the primary cell type that interacts with cells in the fibrotic scar to seal off the lesion core, any genetic manipulation in astrocytes using the Glast-based transgene would confound the interpretation on the role of fibrotic scar-forming cells. Could any leaky Cre recombination in astrocytes reduce astrocytic scarring, potentially leading to the observed axonal and behavioral changes? This possibility remains to be tested with a non-Glast promoter based fate mapping strategy. One challenge is that commonly used markers such as PDGFR $\beta$ are not unique to type A pericytes whereas genetic fate mapping studies critically rely on the specific recombinase transgene used. As illustrated recently, both the injury type and the specific Cre transgenic line used can substantially impact the outcome of such fate mapping studies [6]. Along this line, how are the type A pericytes related to the perivascular fibroblasts described by others as a major source for fibrotic scarring using a collagen1a 1-GFP mouse line [7]? Could they be the same or overlapping cell type(s)? Before further fate mapping studies are conducted using different Cre lines to validate these findings, it might be better to simply refer to these fibrotic scar-forming cells collectively as stromal cells. 
In their previous study using the same Glast promoter-based system [2], the authors reported a protective role of type A pericytes in sealing off the lesion core after spinal cord injury, akin to the well established protective role of reactive astrocytes. Hence, inhibiting fibrotic scarring by blocking type A pericyte proliferation had been anticipated to exert a negative consequence on axonal preservation at the injury site, which will likely worsen functional recovery. On the contrary, the current study describes a positive impact of reducing type A pericyte-derived fibrotic scarring in axon regeneration [1]. The authors still observed detrimental effects of severely inhibiting fibrotic scarring in a subset of animals that exhibited a high degree of recombination (failure to seal off the injury site, leaving a "hole"), replicating their finding previously reported. By stratifying the experimental mice into a high and a medium recombination group, the authors were able to separate the beneficial effect in the medium recombination group from the detrimental effect in the high recombination group. Such grouping is useful in dissecting mechanisms and formulating new hypotheses. Meanwhile, future studies are required to confirm these findings with a prospective experimental design with predictable outcomes.

Recent advances in neuron intrinsic control of axon regeneration accompanied a general realization in the field that targeting neuron extrinsic factors alone is insufficient to elicit robust regeneration especially for axonal tracts refractory to regeneration such as the corticospinal tract [8]. The study by Dias and colleagues renews the debate whether manipulating the CNS environment alone can promote significant regeneration, although such cellular manipulation differs drastically from targeting specific growth inhibitory molecules. As with any significant findings with high importance, the results await validation across labs and experimental conditions before clinical translation [9], especially using a model in which any sparing of the axonal tract is ruled out [10].

\section{Acknowledgments}

We thank Michael Sofroniew for helpful discussion on the injury scar. Supported by grants from NIH/NINSD (NS093055, NS047101), Wings for Life and Craig H. Neilsen foundations.

\section{References}

1. Dias DO, et al. Reducing Pericyte-Derived Scarring Promotes Recovery after Spinal Cord Injury. Cell. 2018; 173:153-165 e122. [PubMed: 29502968]

2. Goritz C, et al. A pericyte origin of spinal cord scar tissue. Science. 2011; 333:238-242. [PubMed: 21737741]

3. Brazda N, Muller HW. Pharmacological modification of the extracellular matrix to promote regeneration of the injured brain and spinal cord. Prog Brain Res. 2009; 175:269-281. [PubMed: 19660662]

4. Ruschel J, et al. Systemic administration of epothilone B promotes axon regeneration after spinal cord injury. Science. 2015; 348:347-352. [PubMed: 25765066]

5. Slezak M, et al. Transgenic mice for conditional gene manipulation in astroglial cells. Glia. 2007; 55:1565-1576. [PubMed: 17823970]

6. Ren Y, et al. Ependymal cell contribution to scar formation after spinal cord injury is minimal, local and dependent on direct ependymal injury. Sci Rep. 2017; 7:41122. [PubMed: 28117356]

7. Soderblom C, et al. Perivascular fibroblasts form the fibrotic scar after contusive spinal cord injury. J Neurosci. 2013; 33:13882-13887. [PubMed: 23966707] 
8. Chen M, Zheng B. Axon plasticity in the mammalian central nervous system after injury. Trends Neurosci. 2014; 37:583-593. [PubMed: 25218468]

9. Steward O, et al. Replication and reproducibility in spinal cord injury research. Exp Neurol. 2012; 233:597-605. [PubMed: 22078756]

10. Steward $\mathrm{O}$, et al. The dorsolateral corticospinal tract in mice: an alternative route for corticospinal input to caudal segments following dorsal column lesions. J Comp Neurol. 2004; 472:463-477. [PubMed: 15065120] 


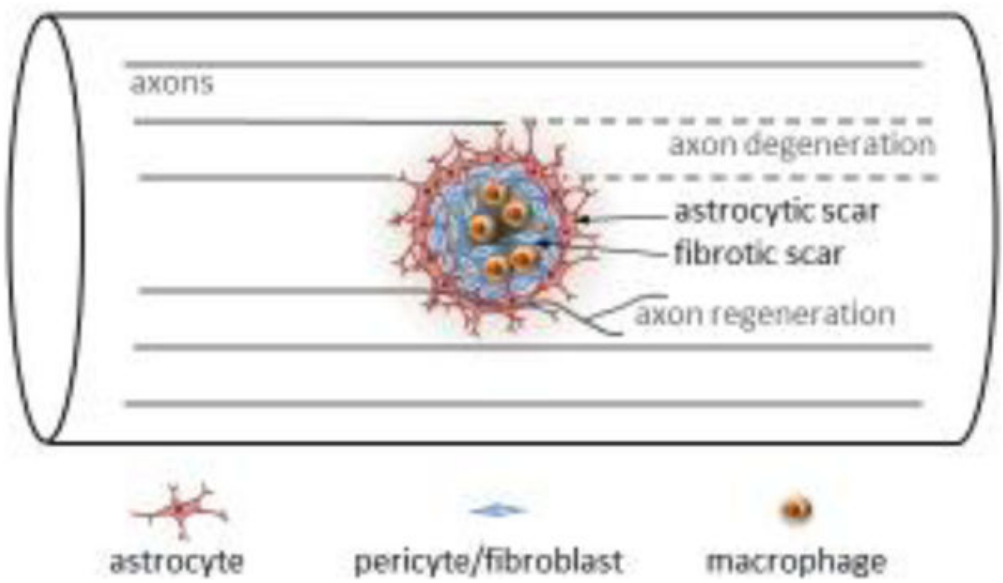

Figure 1.

After spinal cord injury, a scar forms at the injury site including a fibrotic component that is encased by an astrocytic component. How the scarring process impacts repair and recovery remains an important question. Dias and colleagues suggest that reducing type A pericytemediated fibrotic scarring improves axon regeneration (which typically does not occur to a significant extent in the mammalian CNS) and functional recovery. 